\title{
Chronic Cerebral Hypoperfusion Induces Alterations of Matrix Metalloproteinase-9 and Angiopoietin-2 Levels in the Rat Hippocampus
}

\author{
Min-Soo Kim ${ }^{1,4,5 \dagger}$, Bo-Ryoung Choi ${ }^{1 \dagger}$, Yong Woo Lee ${ }^{2}$, Dong-Hee Kim ${ }^{1}$, \\ Ye Sun Han ${ }^{3}$, Won Kyung Jeon ${ }^{4,5 *}$ and Jung-Soo Han ${ }^{1 *}$ \\ ${ }^{1}$ Department of Biological Sciences, Konkuk University, Seoul 05029, Korea, ${ }^{2}$ Department of Biomedical Sciences and \\ Pathobiology, School of Biomedical Engineering and Sciences, Virginia Tech, Virginia 24061, USA, ${ }^{3}$ Department of Advanced \\ Technology Fusion, Konkuk University, Seoul 05029, ${ }^{4}$ Herbal Medicine Research Division, Korea Institute of Oriental \\ Medicine, Daejeon 34054, ${ }^{5}$ Convergence Research Center for Diagnosis, Treatment and Care System of Dementia, Korea \\ Institute of Science and Technology, Seoul 02792, Korea
}

\begin{abstract}
Angiogenic factors contribute to cerebral angiogenesis following cerebral hypoperfusion, and understanding these temporal changes is essential to developing effective treatments. The present study examined temporal alterations in angiogenesis-related matrix metalloproteinase-9 (MMP-9) and angiopoietin-2 (ANG-2) expression in the hippocampus following bilateral common carotid artery occlusion (BCCAo). Male Wistar rats (12 weeks of age) were randomly assigned to sham-operated control or experimental groups, and expression levels of MMP-9 and ANG-2 were assessed after BCCAo (1 week, 4 weeks, and 8 weeks), using western blotting. Protein expression increased 1 week after BCCAo and returned to control levels at 4 and 8 weeks. In addition, immunofluorescence staining demonstrated that the MMP-9- and ANG-2-positive signals were primarily observed in the NeuN-positive neurons with very little labeling in non-neuronal cells and no labeling in endothelial cells. In addition, these cellular locations of MMP-9- and ANG-2-positive signals were not altered over time following BCCAo. Other angiogenic factors such as vascular endothelial growth factor and hypoxia-inducible factor did not differ from controls at 1 week; however, expression of both factors increased at 4 and 8 weeks in the BCCAo group compared to the control group. Our findings increase understanding of alterations in angiogenic factors during the progression of cerebral angiogenesis and are relevant to developing effective temporally based therapeutic strategies for chronic cerebral hypoperfusion-associated neurological disorders such as vascular dementia.
\end{abstract}

Key words: BCCAo, Angiogenesis, Angiopoietin-2, Matrix metalloproteinase-9, Vascular dementia

Received March 12,2018, Revised August 3,2018,

Accepted August 3,2018

* To whom correspondence should be addressed.

Jung-Soo Han, TEL: 82-2-450-3293, FAX: 82-2-3436-5432

e-mail: jshan06@konkuk.ac.kr

Won Kyung Jeon,TEL: 82-42-868-9505, FAX: 82-42-861-5800

e-mail:wkjeon@kiom.re.kr

These authors contributed equally.

\section{INTRODUCTION}

Angiogenesis is a complex process involved in the formation and interaction of multiple vascular regulating factors $[1,2]$. Hypoperfusion, such as that occurring with hypoxia and ischemia, initiates repair process via hypoxia-inducible factor-1 (HIF-1). In addition, expression of angiopoietin-2 (ANG-2) is up-regulated at sites of 
vascular remodeling following hypoxia [3] and lead to an induction of angiogenesis, together with vascular endothelial growth factor (VEGF) [4]. Furthermore, numerous studies have revealed that angiogenesis-related factors are expressed during the recovery phase following hypoperfusion. For example, matrix metalloproteinase-9 (MMP-9) is increased in peri-infarct cortex at 7 14 d after the initial occlusion and exerts beneficial effects by cleaving the extracellular matrix and modulating the intracellular microenvironment [5]. However, these studies focus on the short-term changes in angiogenic factors following initial occlusion, without consideration of long-term effects.

Several animal models have been used to study the effects of hypoperfusion on the brain. Permanent bilateral common carotid artery occlusion (BCCAo) induces chronic cerebral hypoperfusion and the resultant hippocampus-dependent memory impairments [6]. Neuro-inflammation and inflammation-related protein expression are also observed in the hippocampus of rats with BCCAo [7, 8]; furthermore, both damage and repair processes are initiated [9]. To compensate for reductions in cerebral blood flow, the blood vessels dilate, capillaries are recruited, and angiogenesis occurs in conjunction with increased VEGF expression [10-12]. Thus, angiogenesis in the brains of animals with BCCAo may contribute to neuronal reorganization and reduced neurological deficits [13]. Therefore, activation of angiogenesis may reduce the extent of BCCAo-induced damage and mitigate cognitive impairments [14]. However, the underlying molecular mechanisms of angiogenesis following BCCAo have not been elucidated. In particular, alterations to angiogenesis-related factors have not been examined over a long-term course.

Expression level of MMP-9 and ANG-2 is increased prior to the other angiogenic factors such as VEGF and Hif-1a. It is expected that MMP-9 and ANG-2 play a major role in initiating angiogenesis though the other factors are also involved in angiogenesis. Therefore, the present experiment examined time-dependent alterations in angiogenesis-related factors in the hippocampus of BCCAo rats. Rats were randomly assigned to either sham-operated control or BCCAo groups; expression of ANG-2, MMP-9, and the angiogenesis-related factors such as VEGF were examined at 1,4 , and 8 weeks following surgery. And distribution and cellular locations of ANG-2- and MMP-9-positive signals was observed in the hippocampus of BCCAo rats, using fluorescent immunohistochemistry.

\section{MATERIALS AND METHODS}

\section{Animals}

Forty-four naïve 10 weeks-old male (300 340 g) Wistar rats (spe- cific pathogen free) were obtained from Orient Co., a representative of Charles River (Gapeung, South Korea). The rats were 10 weeks of age at the time of arrival and were maintained for 1 week prior to experiments. They were housed individually, in a climatecontrolled vivarium on a 12:12 h light-dark cycle (lights on, 07:00 to $19: 00)$, with controlled temperature $\left(22 \pm 1^{\circ} \mathrm{C}\right)$ and humidity $(50 \pm 10 \%)$. Food and water were available ad libitum. Experiments were conducted in compliance with Konkuk University's Council Directive for the use and care of laboratory animals. Each rat was randomly assigned to the sham-operated control group (8 weeks after the sham-operated surgery) or 1 of the 3 BCCAo groups with different recovery durations ( 1 week, 4 weeks, and 8 weeks; see Fig. 1A).

\section{Surgery}

BCCAo surgeries were performed as previously described [7]. Briefly, rats underwent either BCCAo or sham surgery under $3 \sim 5 \%$ isoflurane/oxygen. Both common carotid arteries were exposed with a midline incision and double ligated with silk sutures. Identical procedures were performed in sham-operated rats, except that the common carotid arteries were not occluded. After all surgical procedures, rats were returned to cages for the recovery period, with free access to food and water.

\section{Brain preparation}

At 1, 4, or 8 weeks following BCCAo surgery, rats were sacrificed by decapitation or perfusion. The hippocampi of decapitated rats were immediately dissected, frozen on dry ice, and stored at $-80^{\circ} \mathrm{C}$ until further experiments. Non-decapitated rats underwent intracardiac perfusion with cold phosphate buffered saline (PBS; $\mathrm{pH} 7.4$ ) followed by $4 \%$ paraformaldehyde in $0.01 \mathrm{M}$ PBS. The brains of perfused rats were fixed in $4 \%$ paraformaldehyde for $3 \mathrm{~d}$, embedded in PBS containing 30\% sucrose until brains sank in the solution, and stored $-80^{\circ} \mathrm{C}$.

\section{Western blot analyses}

Tissue lysate preparation and western blotting occurred as follows. Hippocampal samples were homogenized in ice-cold lysis buffer (20 mM Tris at pH 7.5, 5\% glycerol, 1.5 mM EDTA, $40 \mathrm{mM}$ $\mathrm{KCl}, 0.5 \mathrm{mM}$ dithiothreitol) with protease inhibitors (Calbiochem, La Jolla, CA, USA). Homogenates were centrifuged at $14,000 \mathrm{~g}$ for $1 \mathrm{~h}$. Supernatants were collected, and protein concentrations were determined using Bradford's method. Proteins were separated on SDS-polyacrylamide gels and transferred to polyvinylidene fluoride membranes. The membranes were blocked with fat-free milk in tris-buffered saline containing tween-20 (TBST). After blocking, membranes were incubated overnight at $4^{\circ} \mathrm{C}$ in blocking solution 
A

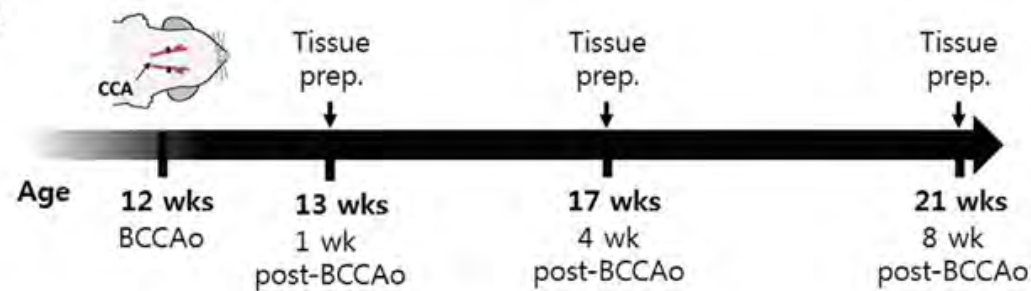

B

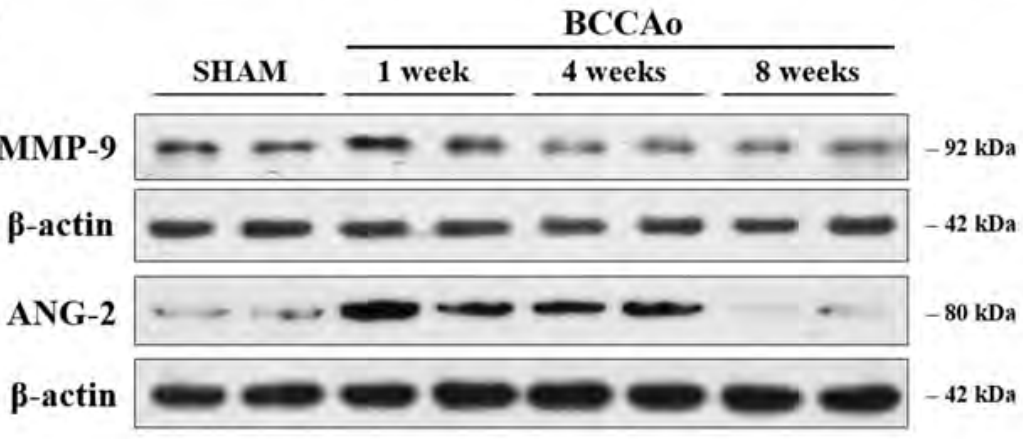

C

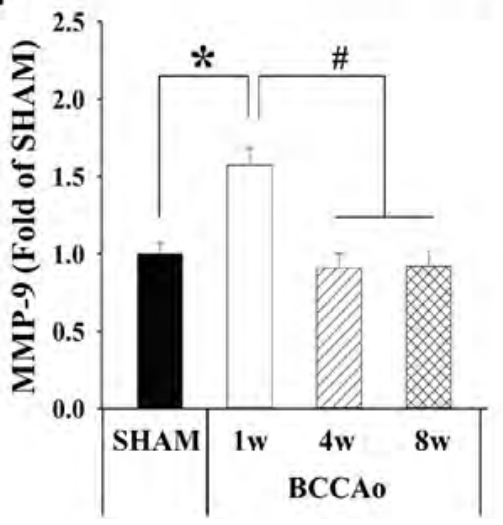

D

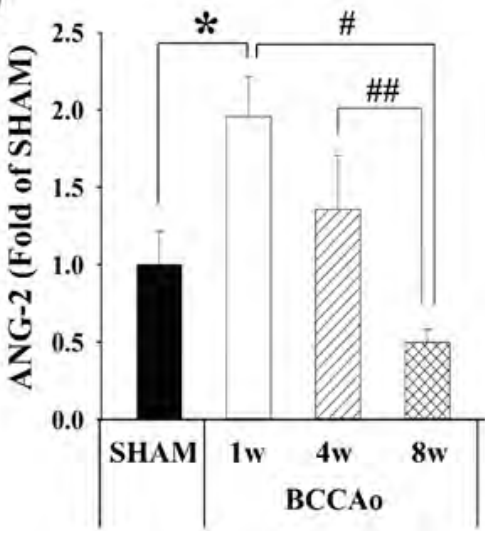

Fig. 1. Changes in hippocampal matrix metalloproteinase-9 (MMP-9) and angiopoietin-2 (ANG2) expression over time following bilateral common carotid artery occlusion (BCCAo). (A) Schematic diagram of experimental procedure. (B) Representative western blot for MMP-9 $(n=4)$ and ANG-2 $(n=5)$. (C) Hippocampal MMP-9 levels were significantly increased at 1 week following BCCAo, compared to controls $\left(^{*}\right)$. Increased MMP-9 returned to control levels at 4 and 8 weeks (\#). (D) Hippocampal ANG2 also increased at 1 week following BCCAo $\left(^{*}\right)$, although its expression levels decreased to control levels at 8 weeks following BCCAo (\#,\#\#). with the addition of primary antibodies. The specific primary antibodies were ANG-2 (1:1000, Millipore, Billerica, MA), COX-2 (1:1000, Santa Cruz, Santa Cruz, CA), Hif-1a (1:250, Santa Cruz), MMP-9 (1:1000, Abcam, Cambridge, MA), TGF- $\beta$ (1:500, Abcam), VEGF (1:500, Santa Cruz), and $\beta$-actin (1:5000, Sigma, St. Louis, MO). The membranes were washed with TBST and incubated for $1 \mathrm{~h}$ at room temperature with anti-rabbit immunoglobulin G (IgG) horseradish peroxidase-conjugated secondary antibody (1:1000, Cell Signaling, Danvers, MA) or anti-mouse IgG antibody (1:2000 or 1:5000, Millipore, Princeton, NJ). Immunoreactions were visualized using an enhanced chemo-luminescence system, and protein bands were scanned and measured using the Image Gauge program (Fuji Film, Tokyo, Japan).

\section{Immunofluorescent staining}

To identify where Ang-2 and MMP-9-positive signals were expressed, ANG-2 and MMP-9 was co-stained with NeuN (neuronal marker), glial fibrillary acidic protein (GFAP, astrocyte marker), CD11b (microglial marker), or Lectin (blood vessel marker). In addition, a single immunofluorescent staining for VEGF was conducted to observe its distribution in the hippocampus. Three sections per animal were washed in PBS with $0.15 \%$ Triton X-100 and then incubated in blocking serum, 5\% normal horse serum in $0.15 \%$ triton with PBS. The sections were then incubated in primary antibody cocktail for 22 hours at room temperature. For primary antibody, mouse anti-NeuN (1:1000, Millipore), mouse antiGFAP (1:500, BD bioscience, San Jose, CA), mouse anti-CD11b (1:500, Bio-Rad, Hercules, CA), rabbit anti-MMP-9 (1:100, Millipore), goat anti-Ang-2 (1:200, Santa Cruz), and FITC-conjugated Tomato-Lectin antibody (1:500, Vector, Burlingame, CA), mouse anti-VEGF antibody (1:500, Santa Cruz) were used. Sections were washed in PBS with $0.15 \%$ Triton X-100 and incubated in the Alexa ${ }^{\circledR}$ fluore conjugated secondary antibody cocktail solution (1:200, Invitrogen, Waltham, MA) for 2 hours at room tempera- 
ture. Stained sections were mounted on resin-coated slides and dried for $30 \mathrm{~min}$. Anti-fade solution (Prolong gold, Invitrogen) was used to maintain the fluorescence.

\section{Statistical analyses}

One-way analysis of variance (ANOVA) was used to analyze protein expression values measured by western blots. Post-hoc analyses (Fisher's least significant difference) then evaluated betweengroups differences if necessary. A p-value $<0.05$ was considered significant. All data are expressed as means \pm standard errors of the mean (SEMs).

\section{RESULTS}

Alterations in MMP-9 and ANG-2 expression levels in the hippocampus following BCCAo

ANOVA indicated a significant effect of group (time point) on hippocampal MMP-9 and ANG-2 expression, as measured by western blots (Fig. 1B; MMP-9, $\mathrm{F}_{(3,12)}=10.719$, p <0.001; ANG-2, $\left.\mathrm{F}_{(3,16)}=6.218, \mathrm{p}<0.01\right)$. Post-hoc analyses for MMP-9 expression revealed that BCCAo rats had significantly higher MMP-9 expression in the hippocampus at 1 week, compared to controls. However, MMP-9 expression returned to control levels at 4 or 8 weeks after BCCAo (Fig. 1C). Hippocampal ANG-2 expression also increased at 1 week after BCCAo; however, expression decreased to control levels at 4 and 8 weeks after BCCAo (Fig. 1D).

\section{Distribution and cellular localization of MMP-9 and ANG-} 2 in the hippocampus of the BCCAo rats

Next, the distribution and cellular localization of MMP-9 and ANG-2 expression was examined in the hippocampus of the BCCAo rats, using triple-labeled fluorescent immunohistochemistry ( $\mathrm{n}=4$ for MMP-9 and ANG-2). As shown in the photomicrography (Fig. 2), MMP-9- and ANG-2-positive signals were mainly located in the NeuN-positive neurons. A very few ANG-2-positive signals were expressed in GFAP-positive glial cells (Fig. 3), but little MMP-

A

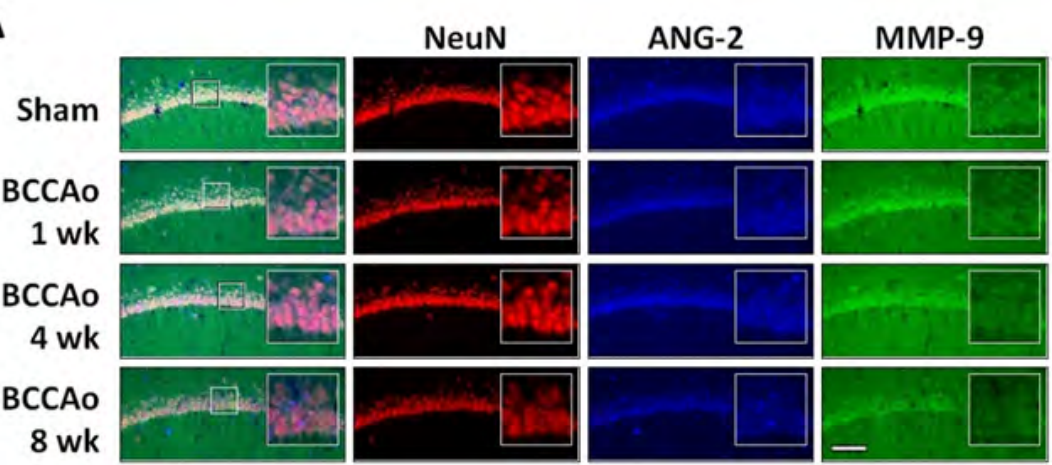

B

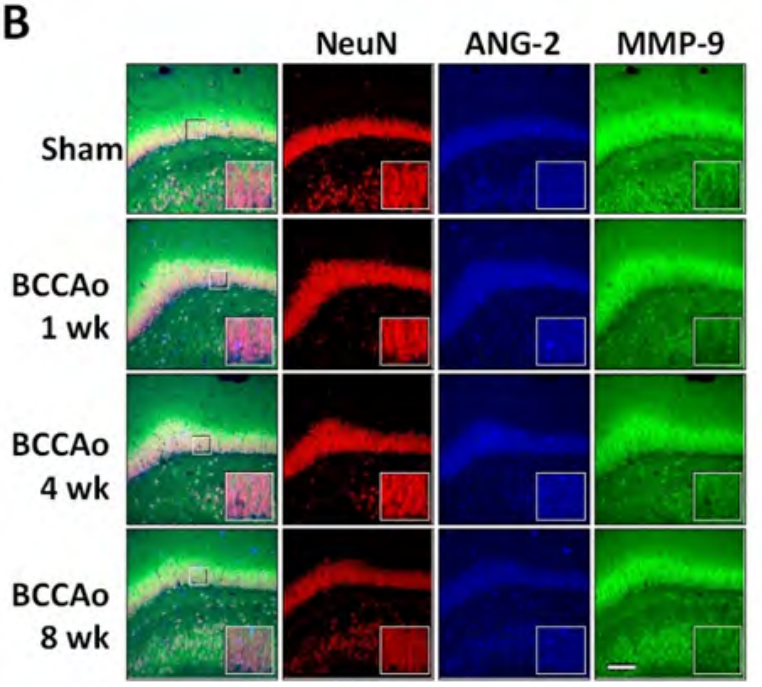

C

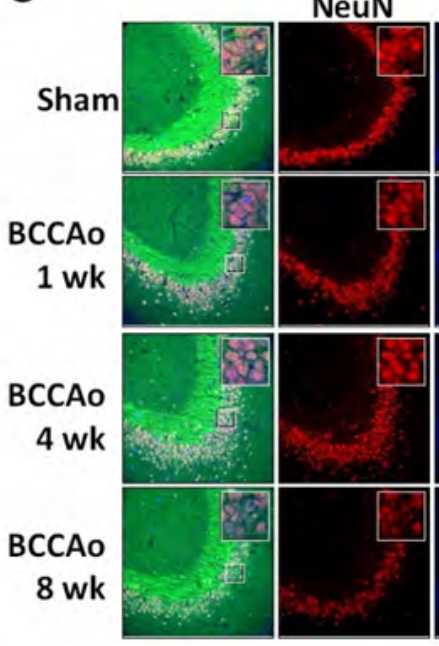

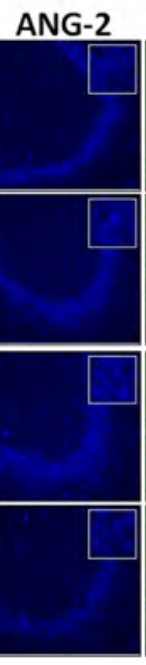

MMP-9

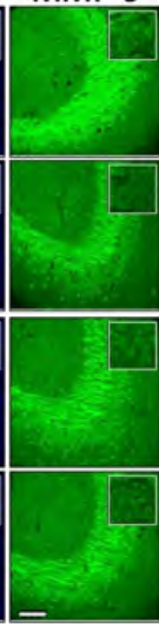

Fig. 2. Distribution and cellular localization of hippocampal angiopoietin-2 (ANG-2) and matrix metalloproteinase-9 (MMP-9) over time following bilateral common carotid artery occlusion (BCCAo) (1 week, 4 weeks, and 8 weeks): Neurons. Most ANG-2 (blue) and MMP-9 (green) labeling was observed in NeuN-positive neurons (red). CA1 (A), dentate gyri (B), and CA3 (C) in the hippocampus. Scale bar, $100 \mu \mathrm{m}$. 
A
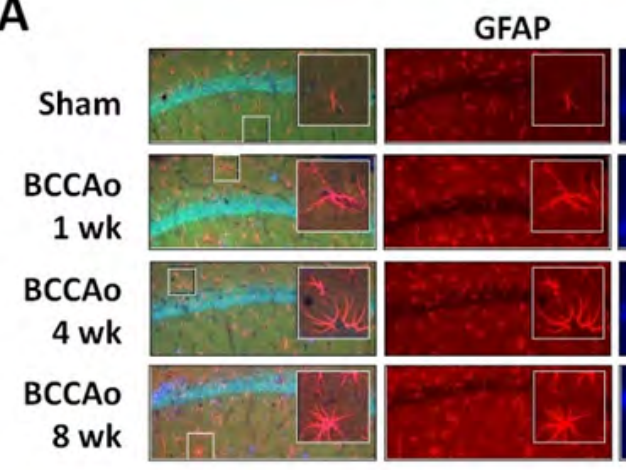

ANG-2
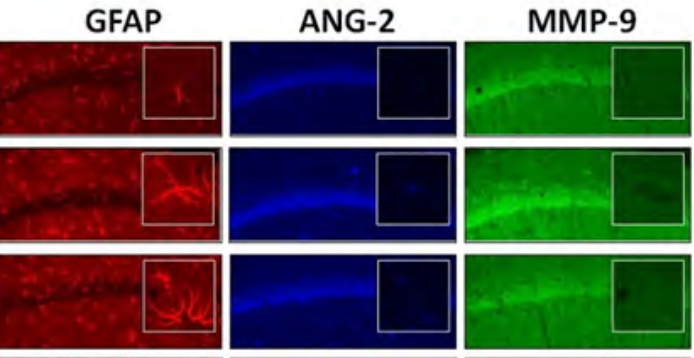

CCAo
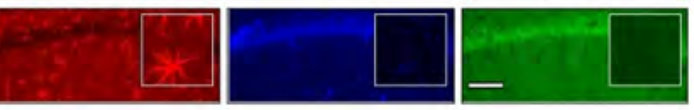

B

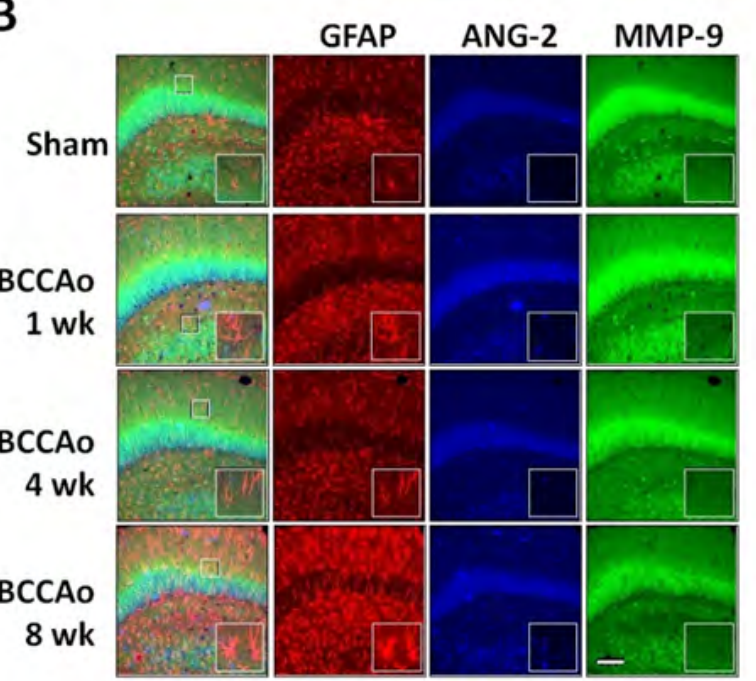

C
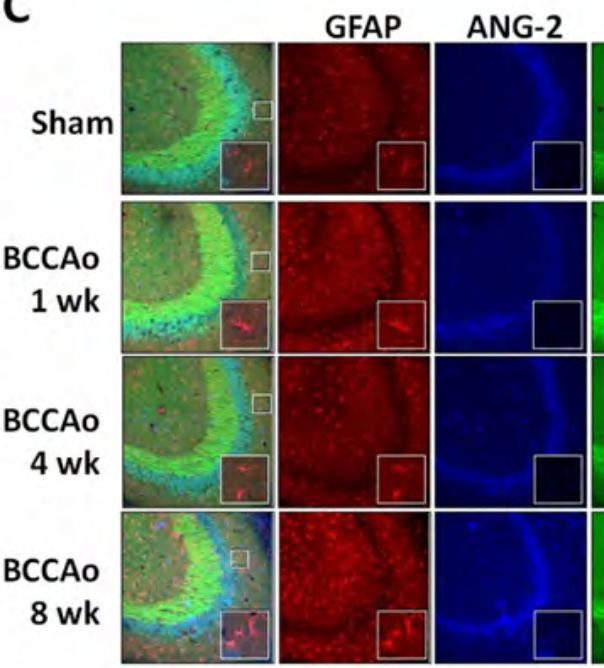

MMP-9

Fig. 3. Distribution and cellular localization of hippocampal angiopoietin-2 (ANG-2) and matrix metalloproteinase-9 (MMP-9) over time following bilateral common carotid artery occlusion (BCCAo) (1 week, 4 weeks, and 8 weeks): glia. A very few ANG-2-positive signals were observed in glial fibrillary acidic protein (GPAP)-positive glial cells, but MMP-9-positive signals were not found in with glial cells. CA1 (A), dentate gyri (B), and CA3 (C) in the hippocampus. Scale bar, $100 \mu \mathrm{m}$.

9-positive signals were found in GFAP-positive glial cells. Both ANG-2 and MMP-9-positive signal was not found in CD11bpositive microglial cells (Fig. 4). We did not find evident changes of ANG-2 and MMP-9 cellular locations over time following BCCAo. These ANG-2 and MMP-9 cellular locations were not altered over time following BCCAo.

\section{MMP-9 and ANG-2 positive signal was not observed in the} blood vessels in the hippocampus of the BCCAo rats

Using triple-labeled fluorescent immunohistochemistry, the expression of MMP-9- and ANG-2-positive signals in blood vessels was evaluated in the hippocampus of the BCCAo rats. We did not find MMP-9- and ANG-2-positive signals located in the Lectinpositive blood vessels over time following BCCAo (Fig. 5).

\section{Alterations in angiogenesis-related factors following BCCAo}

Expression of angiogenesis-related factors previously reported to change in response to chronic hypoperfusion or hypoxia were also measured over time following BCCAo. Hippocampal vascular endothelial growth factor (VEGF) levels were measured using western blots and fluorescent immunohistochemistry. One-way ANOVA demonstrated a significant group effect $\left(\mathrm{F}_{(3,16)}=5.495\right.$, $\mathrm{p}<0.01)$. Subsequent post-hoc analyses indicated that hippocampal VEGF levels were significantly increased at 4 weeks and 8 weeks after BCCAo, compared to the control group (Fig. 6B). Furthermore, as shown in the Fig. 6C, the expression pattern of VEGF in the hippocampal subregions was similar to our findings using western blotting. Expression levels of other angiogenesis-related factors such as hypoxia-inducible factor- $1 \alpha$ (Hif- $1 \alpha$ ) were also altered over time following BCCAo. One-way ANOVA revealed a group effect $\left(\mathrm{F}_{(3,12)}=9.055, \mathrm{p}<0.05\right)$. Subsequent post-hoc analyses indicated that hippocampal Hif-1a significantly increased at 4 weeks and 8 weeks following BCCAo, compared to controls (Fig. $6 \mathrm{D})$. 
A

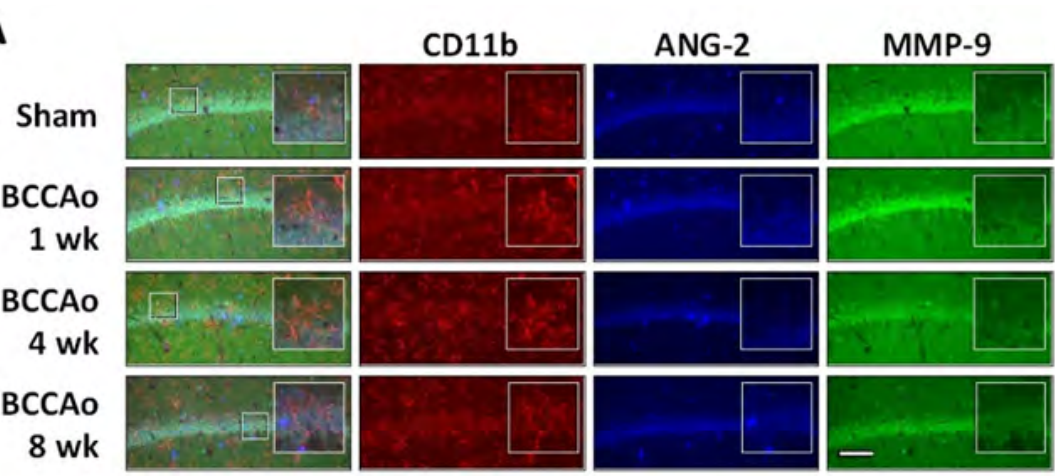

B

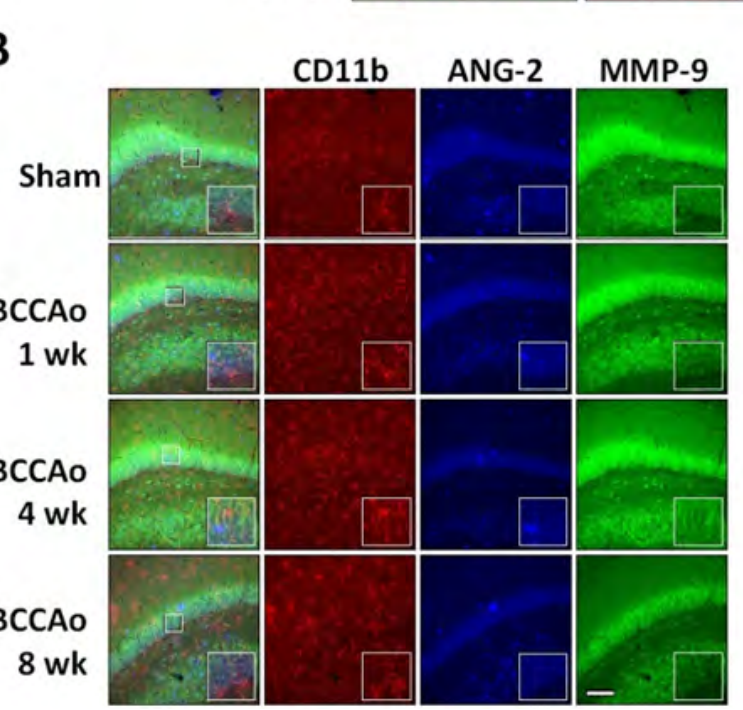

C

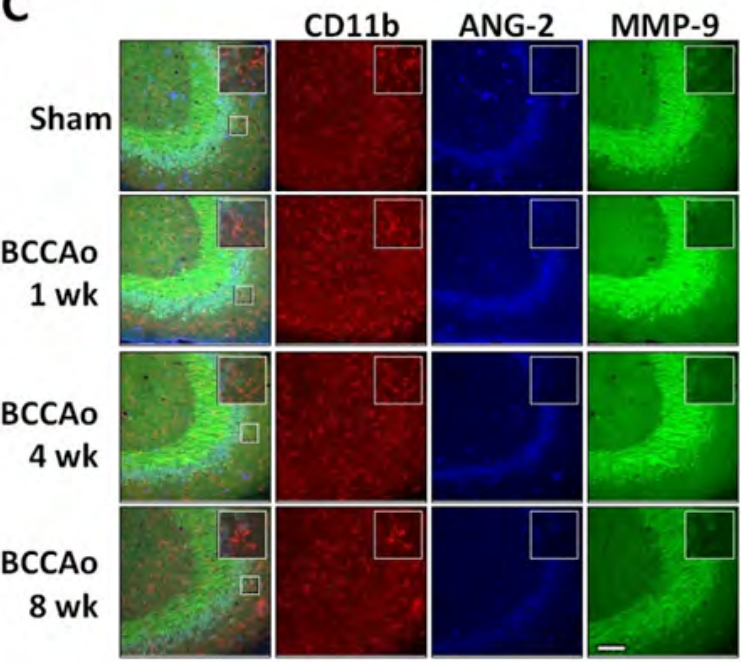

Fig. 4. Distribution and cellular localization of hippocampal angiopoietin-2 (ANG-2) and matrix metalloproteinase-9 (MMP-9) over time following bilateral common carotid artery occlusion (BCCAo) (1 week, 4 weeks, and 8 weeks): Microglia. Both ANG-2 and MMP-9-positive signals were not found in CD11b-positive microglial cells. CA1 (A), dentate gyri (B), and CA3 (C) in the hippocampus. Scale bar, $100 \mu \mathrm{m}$.

Expression levels of angiogenesis-related cytokines such as cyclooxygenase-2 (COX-2) and transforming growth factor-beta (TGF- $\beta$ ) were measured over time following BCCAo, using western blots. One-way ANOVA revealed significant group effects: COX-2, $\mathrm{F}_{(3,12)}=5.913, \mathrm{p}<0.01$; TGF- $\beta, \mathrm{F}_{(3,12)}=4.915, \mathrm{p}<0.05$. Posthoc analyses indicated that expression increased at 1 and 4 weeks following BCCAo, but returned to control levels at 8 weeks (Fig. $6 \mathrm{E}, \mathrm{F})$.

\section{DISCUSSION}

BCCAo-induced chronic cerebral hypoperfusion leads to neuroinflammation and white matter damage, resulting in hippocampus-dependent memory impairments [6]. Furthermore, in response to chronic cerebral hypoperfusion, both neurodegenerative cascades and neuroprotective or repair mechanisms are initiated. To compensate for reduced cerebral blood flow, angiogenic factors are activated, thereby generating new microcapillaries.
Previous studies have demonstrated that regional cerebral blood flow decreases to $33 \% \sim 45 \%$ of control levels in the cortex at $2 \mathrm{~d}$ following BCCAo, and recovers to baseline values by 8 weeks postsurgery [15].

Angiogenesis is well-characterized in a stroke model of focal ischemia via middle cerebral artery occlusion [16, 17]. In contrast, angiogenesis has not been clearly established during global ischemia resulting from chronic BCCAo. Moreover, these studies have focused on short-term changes in angiogenic factors following initial occlusion, rather than on long-term changes. Therefore, the present study measured several angiogenesis-related factors over relatively long-time periods (up to 8 weeks) in the hippocampus of rats with BCCAo. Specifically, MMP-9 and ANG-2, which may be up-regulated following hypoxia or hypoperfusion $[3,5]$, were measured in the hippocampus and their cellular locations were examined at 1 week, 4 weeks, and 8 weeks following BCCAo.

MMPs enhance angiogenesis by cleaving extracellular matrix $[9,18]$. In particular, MMP-9 enhances angiogenesis by cleaving 
A

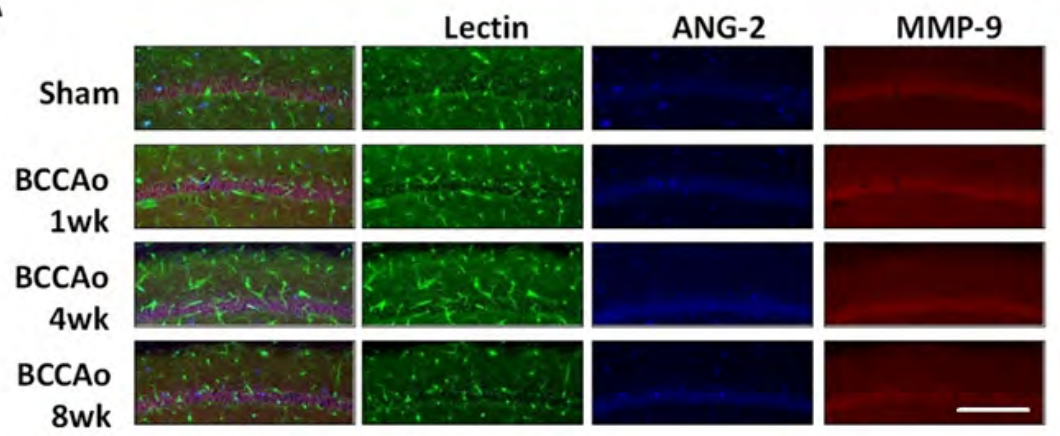

B
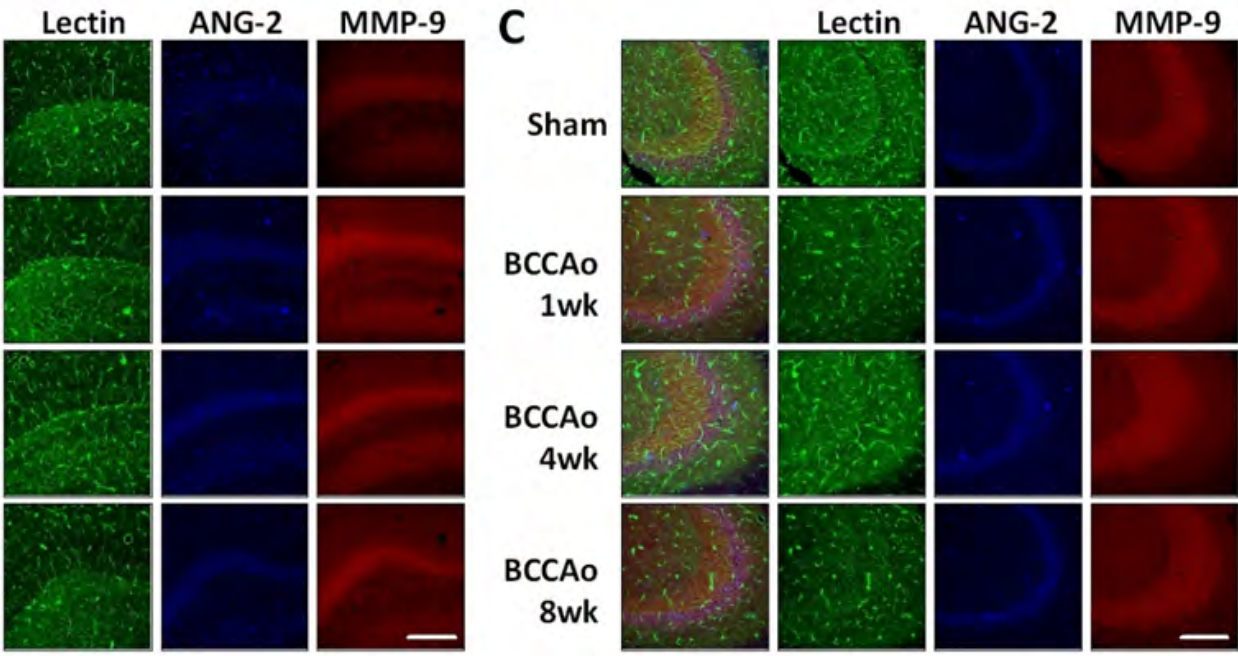

Fig. 5. Hippocampal angiopoietin-2 (ANG-2) and matrix metalloproteinase-9 (MMP-9) labeling was not observed in the enthothelial cells stained with lectin. CA1 (A), dentate gyri (B), and CA3 (C) in the hippocampus. Scale bar, $200 \mu \mathrm{m}$.

endothelial cell-to-cell adhesion [2]. Previous reports indicate that MMP-9 is upregulated and diffuses throughout the ischemic cortex [5, 19]. In the present study, hippocampal MMP-9 levels increased at 1 week following BCCAo, although expression had returned to baseline at 4 and 8 weeks. MMP- 9 was primarily located in neurons, rather than in astrocytes and microglia. In addition, ANG-2 mediates vascular remodeling [20] and alters blood vessel responses to angiogenic stimuli during quiescence states [14]. A recent study found that blocking ANG-2 inhibits angiogenesis in the retinal vasculature of newborn mice [21]. The present results demonstrate that altered ANG-2 expression over time, as well as the cellular locations in the hippocampus of BCCAo rats, were similar to those for MMP-9; however, the results of fluorescent immunohistochemistry did not show significant alterations over time. Studies using a stroke model have observed MMP-9 and ANG-2 expression in astrocytes [5, 22]. However, these expression in astrocyte has not been observed in the hippocampus with chronic BCCAo, although a very few ANG-2 signals were observed in the astrocytes. Therefore, our findings suggest that MMP-9 and ANG-2 are activated as components of different mechanisms for initiating angiogenesis during chronic cerebral hypoperfusion from those involved in the stroke. The present study also examined whether ANG-2 and MMP-9 was expressed in the blood vessels using Lectin as a marker of endothelial cell [23]. However, Lectin-positive signals were not co-localized with MMP-9- or ANG-2-positive signals.

The cellular locations of ANG-2 and MMP-9-positive signals after BCCAo have not yet been reported. MMP-9 is reported to be predominantly but not exclusively expressed in neurons in the rat hippocampus [24], and ANG-2 is produced by endothelial cells and expressed at sites of endothelial cell activation at basal state [25]. Several studies have reported that MMP-9-positive signals were seen in microglia, neuron, and astrocytes after stroke or hypoxia-ischemia $[5,26]$. Another study using a stroke model has reported that ANG-2 was expressed in astrocytes of subventricular zone progenitor cells [22]. On the other hand, our findings showed that ANG-2 and MMP-9 were primarily located in neurons following BCCAo. These results imply that mechanisms of angiogenesis by angiogenic factors during chronic cerebral hypoperfusion might be different from angiogenesis during stroke. 

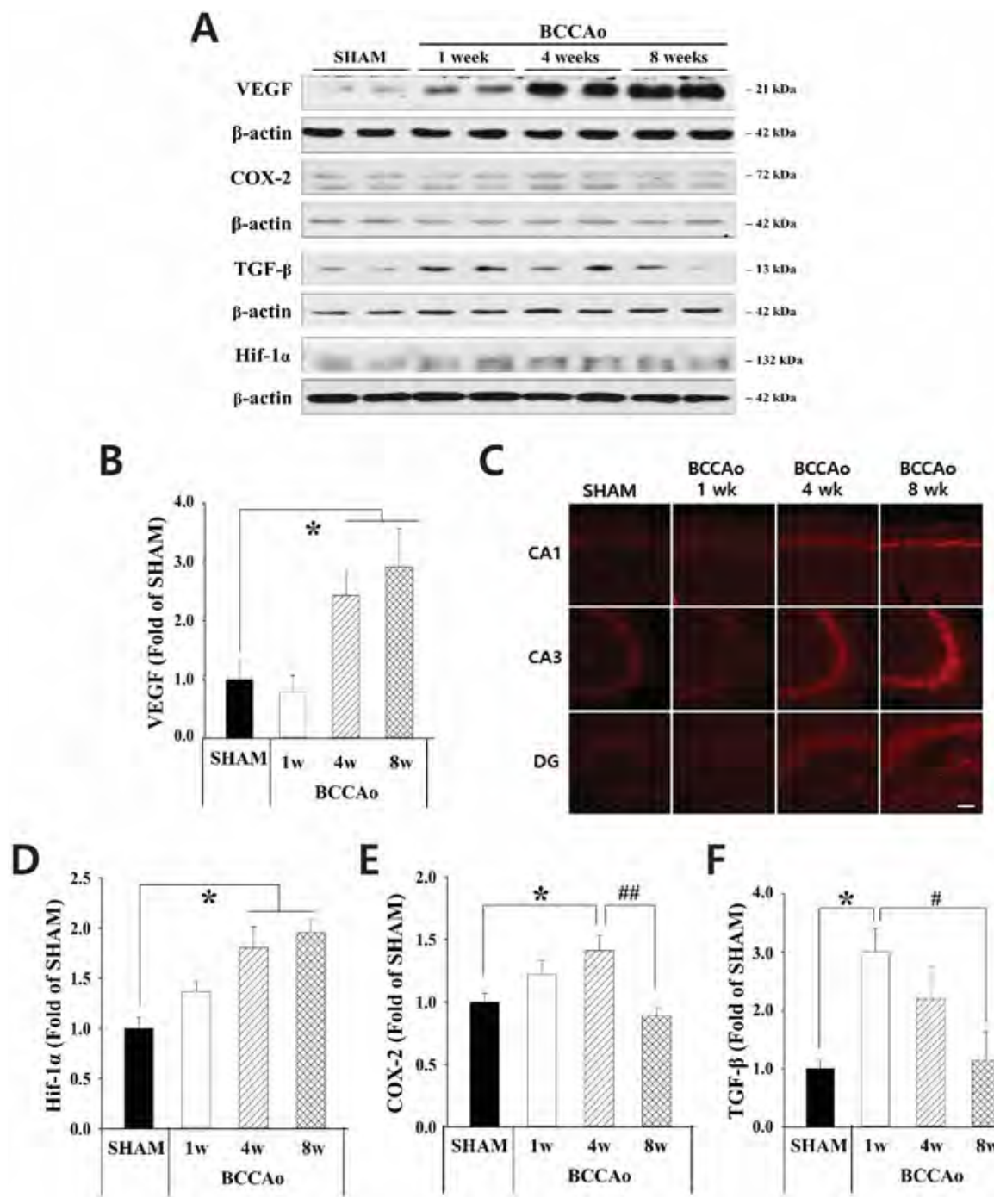

F

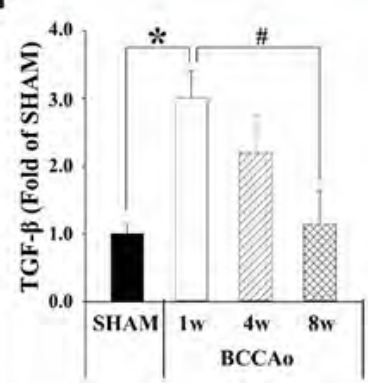

Fig. 6. Alterations in angiogenesis-related factors and cytokines over time following bilateral common carotid artery occlusion (BCCAo). (A) Western blots of hippocampal vascular endothelial growth factor (VEGF, $n=5)$, cyclooxygenase- 2 (COX-2, $n=4)$, transforming growth factor $\beta$ (TGF- $\beta, n=4$ ), and hypoxia inducible factor- $1 \alpha$ (Hif- $1 \alpha, n=4)$. (B) Hippocampal VEGF levels by quantified results of western blots significantly increased at 4 and 8 weeks $\left(^{*}\right)$ after BCCAo, compared to controls. (C) Fluorescent immunohistochemistry of hippocampal VEGF. (D) Hippocampal Hif-1 a significantly increased at 4 weeks and 8 weeks following BCCAo compared to controls ${ }^{*}$ ). (E, F) Hippocampal COX-2 and TGF- $\beta$ increased at 1 and 4 weeks following BCCAo $\left(^{*}\right)$, although expression returned to control levels by 8 weeks (\#,\#). Scale bar, $100 \mu \mathrm{m}$.
However further studies are necessary to explore the mechanisms underlying functional recovery by angiogenic factors following BCCAo.

ANG-2 expression might initiate angiogenesis by reducing vascular stability and play a major role in vascular angioplasticity [14]. In addition, MMP-9 induce angiogenesis by regulating pericyte detachment from vessels [2]. After the physical barrier is removed, downstream of angiogenic signaling is activated under the influence of the Hif-1a with its regulated gene VEGF. Previous study has demonstrated that vascular growth was preceded by vascular regression in a stroke model, correlating with an alteration of VEGF/ANG-2 balance. Therefore, these reports and our present results suggest that MMP-9/ANG-2 and VEGF/HIF-1 $\alpha$ are triggered after ischemia over different recovery periods [27]. Specifically, expression levels of MMP-9 and ANG-2 were increased prior to the other angiogenic factors such as VEGF and Hif-1 $\alpha$ following BCCAo. These results imply that MMP-9 and ANG-2 play a major role in initiating angiogenesis though the other factors are also needed to process angiogenesis. Thus, regulation of MMP-9 and ANG-2 at early point following chronic cerebral hypoperfusion might be important for functional recovery.

Furthermore, VEGF contributes to microvessel sprouting [18] and mediates neovascularization [17]. VEGF promotes blood vessel growth, as well as synapse formation and maturation [28]. Furthermore, previous studies have reported that combined ANG2 and VEGF treatment facilitates angiogenesis [29]; therefore, hippocampal VEGF was measured over time following BCCAo. Interestingly, hippocampal VEGF expression did not significantly differ in BCCAo rats compared to controls at 1 week, although VEGF was significantly increased at 4 weeks and 8 weeks. Moreover, HIF- $1 \alpha$ is an angiogenesis regulator following VEGF induction [30] and increases with chronic cerebral hypoperfusion [31]. Several studies have demonstrated that VEGF is regulated by MMP-9, which may mediate the angiogenic switch [32]. Our 
results therefore suggest that increased MMP-9 following BCCAo may contribute to upregulated VEGF expression. In addition, HIF1a induces VEGF mRNA expression in response to hypoxia [33]. We observed that expression of Hif- $1 \alpha$ increased gradually following BCCAo, which is consistent with a previous study [31]. Finally, ANG-2 is also activated by inflammatory factors such as TGF- $\beta$ and COX-2 $[14,20,22]$. In the present study, hippocampal expression of these inflammatory factors gradually increased following BCCAo.

The present study showed that neuronal MMP-9 and ANG-2 are released in the short-term phase, whereas VEGF and VEGFrelated factor expression increase in the long-term phase following BCCAo. These findings may result from active regulation of angiogenesis-related factors at different time points following BCCAo. Considering that angiogenesis contributes to functional recovery during the chronic post-stroke phase [14, 34], regulating angiogenesis-related factors at the appropriate points in recovery may be useful for treating the pathology associated with vascular dementia and other neurovascular diseases. Therefore, these findings suggest that ANG-2 and MMP-9 are released from neurons in response to cerebral hypoperfusion, and therefore may contribute to angiogenesis.

\section{ACKNOWLEDGEMENTS}

This work was funded by the R \& D Convergence Program of the Republic of Korea National Research Council of Science \& Technology (grant numbers CRC-15-04-KIST, G15120, G16230, K16850 and K17850) and the Korea Health Technology R \& D Project, through the Korea Health Industry Development Institute (KHIDI), which is funded by the Ministry of Health \& Welfare, Republic of Korea (grant number HI15C1540).

\section{REFERENCES}

1. Benderro GF, Sun X, Kuang Y, Lamanna JC (2012) Decreased VEGF expression and microvascular density, but increased HIF- 1 and $2 \alpha$ accumulation and EPO expression in chronic moderate hyperoxia in the mouse brain. Brain Res 1471:4655.

2. Rundhaug JE (2005) Matrix metalloproteinases and angiogenesis. J Cell Mol Med 9:267-285.

3. Simon MP, Tournaire R, Pouyssegur J (2008) The angiopoietin-2 gene of endothelial cells is up-regulated in hypoxia by a HIF binding site located in its first intron and by the central factors GATA-2 and Ets-1. J Cell Physiol 217:809-818.

4. Etoh T, Inoue H, Tanaka S, Barnard GF, Kitano S, Mori M
(2001) Angiopoietin-2 is related to tumor angiogenesis in gastric carcinoma: possible in vivo regulation via induction of proteases. Cancer Res 61:2145-2153.

5. Zhao BQ, Wang S, Kim HY, Storrie H, Rosen BR, Mooney DJ, Wang X, Lo EH (2006) Role of matrix metalloproteinases in delayed cortical responses after stroke. Nat Med 12:441-445.

6. Farkas E, Luiten PG, Bari F (2007) Permanent, bilateral common carotid artery occlusion in the rat: a model for chronic cerebral hypoperfusion-related neurodegenerative diseases. Brain Res Rev 54:162-180.

7. Choi BR, Lee SR, Han JS, Woo SK, Kim KM, Choi DH, Kwon KJ, Han SH, Shin CY, Lee J, Chung CS, Lee SR, Kim HY (2011) Synergistic memory impairment through the interaction of chronic cerebral hypoperfusion and amlyloid toxicity in a rat model. Stroke 42:2595-2604.

8. Choi BR, Kwon KJ, Park SH, Jeon WK, Han SH, Kim HY, Han JS (2011) Alternations of septal-hippocampal system in the adult wistar rat with spatial memory impairments induced by chronic cerebral hypoperfusion. Exp Neurobiol 20:92-99.

9. Rosenberg GA (2009) Matrix metalloproteinases and their multiple roles in neurodegenerative diseases. Lancet Neurol 8:205-216.

10. de Wilde MC, Farkas E, Gerrits M, Kiliaan AJ, Luiten PG (2002) The effect of $n-3$ polyunsaturated fatty acid-rich diets on cognitive and cerebrovascular parameters in chronic cerebral hypoperfusion. Brain Res 947:166-173.

11. Ohtaki H, Fujimoto T, Sato T, Kishimoto K, Fujimoto M, Moriya M, Shioda S (2006) Progressive expression of vascular endothelial growth factor (VEGF) and angiogenesis after chronic ischemic hypoperfusion in rat. Acta Neurochir Suppl (Wien) 96:283-287.

12. Choy M, Ganesan V, Thomas DL, Thornton JS, Proctor E, King MD, van der Weerd L, Gadian DG, Lythgoe MF (2006) The chronic vascular and haemodynamic response after permanent bilateral common carotid occlusion in newborn and adult rats. J Cereb Blood Flow Metab 26:1066-1075.

13. Slevin M, Kumar P, Gaffney J, Kumar S, Krupinski J (2006) Can angiogenesis be exploited to improve stroke outcome? Mechanisms and therapeutic potential. Clin Sci (Lond) 111:171-183.

14. Liu J, Wang Y, Akamatsu Y, Lee CC, Stetler RA, Lawton MT, Yang GY (2014) Vascular remodeling after ischemic stroke: mechanisms and therapeutic potentials. Prog Neurobiol 115:138-156.

15. Otori T, Katsumata T, Muramatsu H, Kashiwagi F, Katayama Y, Terashi A (2003) Long-term measurement of cerebral 
blood flow and metabolism in a rat chronic hypoperfusion model. Clin Exp Pharmacol Physiol 30:266-272.

16. Zan LK, Song YJ, Teng GX, Li H, Liu W, Jia Y, Zhou M, Sun YL, Qi JP (2011) Expression and function of vascular endothelial growth factor and angiopoietins in rat brain after cerebral ischemia. Zhonghua Bing Li Xue Za Zhi 40:834-839.

17. Lennmyr F, Ata KA, Funa K, Olsson Y, Terént A (1998) Expression of vascular endothelial growth factor (VEGF) and its receptors (Flt-1 and Flk-1) following permanent and transient occlusion of the middle cerebral artery in the rat. J Neuropathol Exp Neurol 57:874-882.

18. Zhu Y, Lee C, Shen F, Du R, Young WL, Yang GY (2005) Angiopoietin-2 facilitates vascular endothelial growth factorinduced angiogenesis in the mature mouse brain. Stroke 36:1533-1537.

19. Rosenberg GA, Cunningham LA, Wallace J, Alexander S, Estrada EY, Grossetete M, Razhagi A, Miller K, Gearing A (2001) Immunohistochemistry of matrix metalloproteinases in reperfusion injury to rat brain: activation of MMP-9 linked to stromelysin- 1 and microglia in cell cultures. Brain Res 893:104-112.

20. Pichiule P, LaManna JC (2002) Angiopoietin-2 and rat brain capillary remodeling during adaptation and deadaptation to prolonged mild hypoxia. J Appl Physiol (1985) 93: 1131-1139.

21. Felcht M, Luck R, Schering A, Seidel P, Srivastava K, Hu J, Bartol A, Kienast Y, Vettel C, Loos EK, Kutschera S, Bartels S, Appak S, Besemfelder E, Terhardt D, Chavakis E, Wieland T, Klein C, Thomas M, Uemura A, Goerdt S, Augustin HG (2012) Angiopoietin-2 differentially regulates angiogenesis through TIE2 and integrin signaling. J Clin Invest 122:19912005.

22. Liu XS, Zhang ZG, Zhang RL, Gregg S, Morris DC, Wang Y, Chopp M (2007) Stroke induces gene profile changes associated with neurogenesis and angiogenesis in adult subventricular zone progenitor cells. J Cereb Blood Flow Metab 27:564574.

23. Roussel F, Dalion J (1988) Lectins as markers of endothelial cells: comparative study between human and animal cells. Lab Anim 22:135-140.

24. Szklarczyk A, Lapinska J, Rylski M, McKay RD, Kaczmarek L
(2002) Matrix metalloproteinase-9 undergoes expression and activation during dendritic remodeling in adult hippocampus. J Neurosci 22:920-930.

25. Hegen A, Koidl S, Weindel K, Marmé D, Augustin HG, Fiedler U (2004) Expression of angiopoietin-2 in endothelial cells is controlled by positive and negative regulatory promoter elements. Arterioscler Thromb Vasc Biol 24:1803-1809.

26. Svedin P, Hagberg H, Sävman K, Zhu C, Mallard C (2007) Matrix metalloproteinase-9 gene knock-out protects the immature brain after cerebral hypoxia-ischemia. J Neurosci 27:1511-1518.

27. Beck H, Acker T, Wiessner C, Allegrini PR, Plate KH (2000) Expression of angiopoietin-1, angiopoietin-2, and tie receptors after middle cerebral artery occlusion in the rat. Am J Pathol 157:1473-1483.

28. Tillo M, Ruhrberg C, Mackenzie F (2012) Emerging roles for semaphorins and VEGFs in synaptogenesis and synaptic plasticity. Cell Adhes Migr 6:541-546.

29. Carmeliet P (2003) Angiogenesis in health and disease. Nat Med 9:653-660.

30. Ndubuizu OI, Tsipis CP, Li A, LaManna JC (2010) Hypoxiainducible factor-1 (HIF-1)-independent microvascular angiogenesis in the aged rat brain. Brain Res 1366:101-109.

31. Yang Y, Zhang J, Liu H, Wang J, Xin J, Deng M (2013) Changes in levels of hypoxia-induced mediators in rat hippocampus during chronic cerebral hypoperfusion. Neurochem Res 38:2433-2439.

32. Hu Q, Chen C, Yan J, Yang X, Shi X, Zhao J, Lei J, Yang L, Wang K, Chen L, Huang H, Han J, Zhang JH, Zhou C (2009) Therapeutic application of gene silencing MMP-9 in a middle cerebral artery occlusion-induced focal ischemia rat model. Exp Neurol 216:35-46.

33. Hirota K (2002) Hypoxia-inducible factor 1, a master transcription factor of cellular hypoxic gene expression. J Anesth 16:150-159.

34. Arai K, Jin G, Navaratna D, Lo EH (2009) Brain angiogenesis in developmental and pathological processes: neurovascular injury and angiogenic recovery after stroke. FEBS J 276:46444652 . 